\title{
Microstructure Characterization of Surface Modified 316L Stainless Steel Using an Automated Electron Diffraction Technique in the TEM
}

\author{
G. Facco, A. Kulovits, J. M. K. Wiezorek \\ Department of Mechanical Engineering and Materials Science, Swanson School of Engineering, University \\ of Pittsburgh, Benedum Hall 323, 3700 O Hara Street, Pittsburgh PA 15261
}

Establishing quantitatively relationships between processing parameters, microstructural metrics (e.g. grain size, internal strain, grain boundary character distributions and texture) and properties of nanoscale polycrystalline aggregate materials requires experimental tools with nanometer spatial resolution that can deliver statistically significant data sets. We use automated systems for acquisition and indexing of diffraction patterns (ASTAR by Nanomegas) in the transmission electron microscope (TEM) to characterize the micro- and nano-scaled structure changes in $316 \mathrm{~L}$ stainless steel in response to linear raking, a novel surface severe plastic deformation process. The system acquires precession electron diffraction patterns to identify crystal orientations of nanoscale volumes. The condensor lens system in the TEM is used to precess the incident beam with a fixed angle (Fig. 1d). The image shift lens system is used to descan the diffraction pattern (DP) to convert the hollow cone DP into a spot pattern, that includes higher order $\mathbf{g}$ - vectors, can be treated kinematically and easily indexed (Fig. 1d). The system uses scans the precessing beam over preselected areas that are several square microns in size providing large data sets. The electron beam probe size determines the spatial resolution limit and the automated indexing enables orientation imaging microscopy (OIM) [1]. A 20nm probe was sufficient to successfully characterize quantitatively the nanoscale refined microstructure of severely plastically deformed 316L stainless steel. Figure 1 compares the microstructure of the 316L material prior to and after raking processes. From SEM images as shown in Fig. 1a we determined an average grain size of $\sim 30 \mu \mathrm{m}$ for the starting material. In comparison the bright field image (BF) in Fig. $1 \mathrm{~b}$ shows a massively refined microstructure after $0^{\circ}$ rake angle processing. Hollow cone dark field analysis (HCDF; not shown here) yielded an average grain size on the order of $2-300 \mathrm{~nm}$. The corresponding diffraction pattern (Fig. 1b) shows little strain storage induced radial broadening but misorientation spread induced angular arching consistent with grain refinement rather than strain storage, indicating that $0^{\circ}$ rake angle processing constitutes severe plastic deformation. $20^{\circ}$ rake angle processing results in storage of large amount of strain (Fig. 1c), indicated by the increased radial broadening in the DP, identifying it as a conventional deformation process. Figure 2 shows an orientation, phase and grain boundary character analysis of the $316 \mathrm{~L}$ prior to and after deformation. Grain sizes obtained by orientation mapping in the TEM are consistent with BF and HCDF image analyses. Phase identification shows little to no martensite formation after the $0^{\circ}$ rake angle processing and a large volume fraction of stress induced martensite after the $20^{\circ}$ rake angle process. Grain boundary character analysis (Fig. 2e) shows a drastic reduction of $\Sigma 3$ grain boundaries after the raking processes.

We acknowledge use of the facilities of the Materials Micro-Characterization Laboratory of the Department of Mechanical Engineering and Materials Science, University of Pittsburgh, and support by a grant from the Nuclear Regulatory Commission, NRC-3809-935 


\section{References}

[1] Palumbo, G et al. Scripta Matallurgica et Materialia (1991) 25: 1775-1780

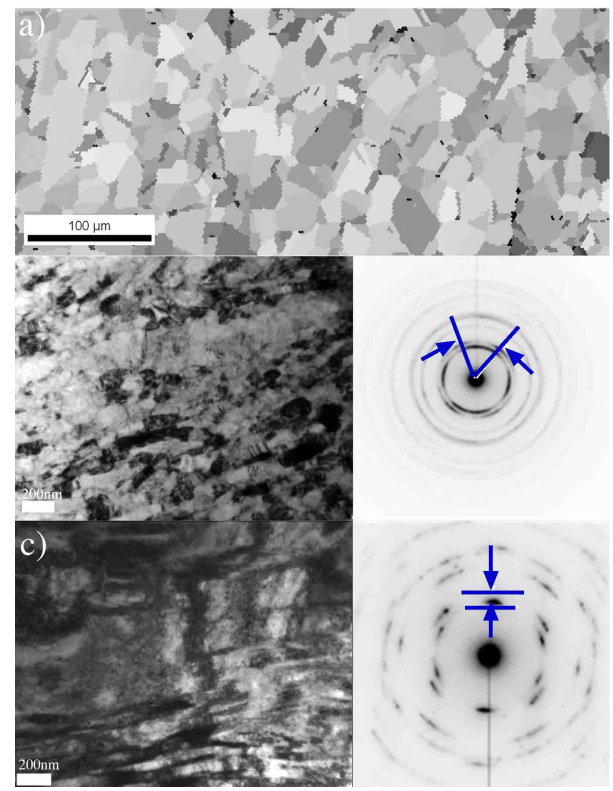

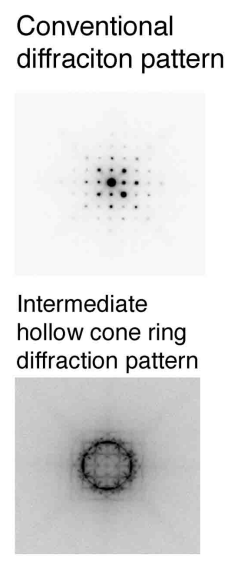

Precession diffraction pattern

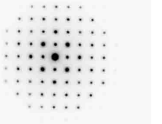

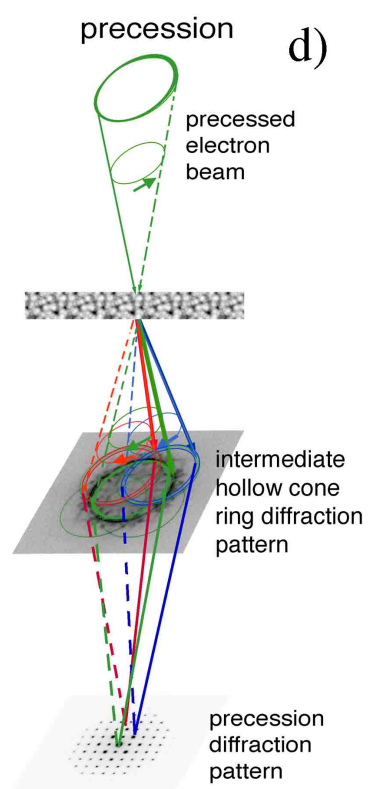

FIG. 1. a) SEM image of the as received microstructure of 316L; b) TEM BF image and corresponding DP after $0^{\circ}$ raking; c) TEM BF image and corresponding DP after $20^{\circ}$ raking; d) Schematic of precession electron diffraction
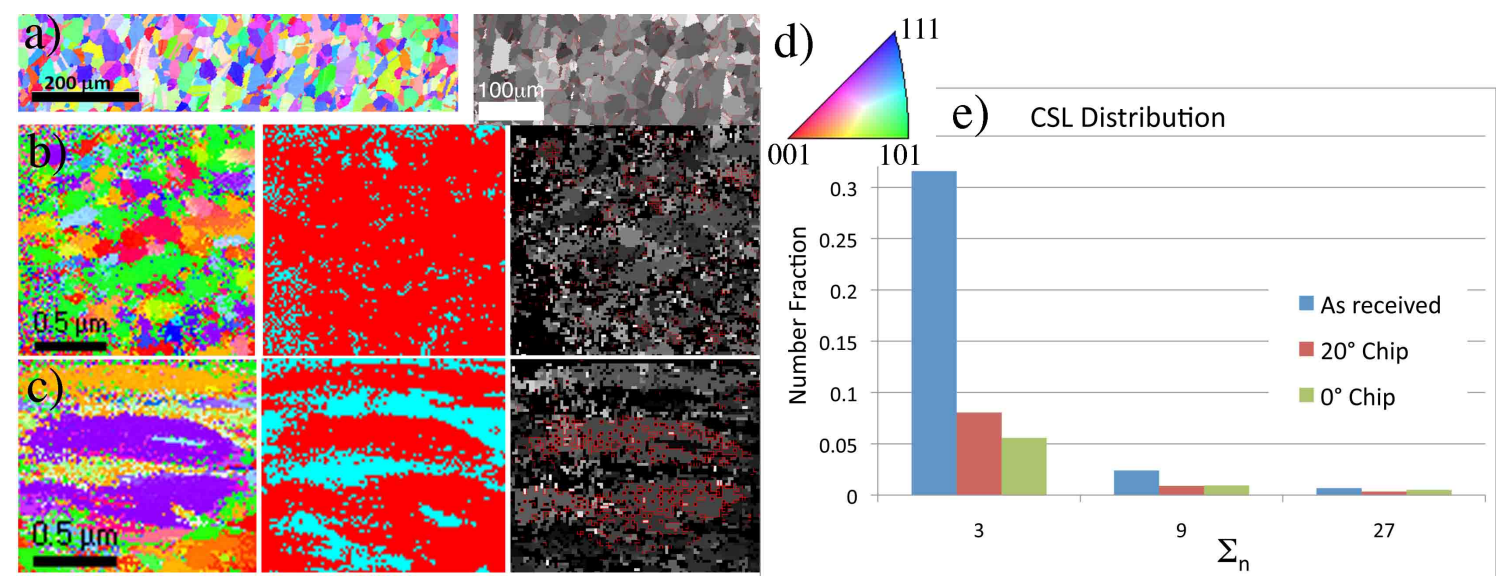

FIG. 2. a) OIM orientation distribution and CSL map of the as received state; b) OIM orientation distribution map, phase map ( $\mathrm{red}=$ austenite, blue $=$ martensite $)$ and CSL map after $0^{\circ}$ raking c) OIM orientation distribution map, phase map ( red = austenite, blue = martensite) and CSL map after $20^{\circ}$ raking; d) orientation distribution map legend; e) Comparison of the change of coincident site lattice boundaries of the as received, $0^{\circ}$ and $20^{\circ}$ raking samples. 